\title{
Sharp lower bounds for Coulomb energy
}

\author{
Jacopo Bellazzini, Marco Ghimenti, and Tohru Ozawa
}

We prove $L^{p}$ lower bounds for Coulomb energy for radially symmetric functions in $\dot{H}^{s}\left(\mathbb{R}^{3}\right)$ with $\frac{1}{2}<s<\frac{3}{2}$. In case $\frac{1}{2}<s \leq 1$ we show that the lower bounds are sharp.

\section{Introduction}

In this paper we prove lower bounds for the Coulomb energy

$$
\iint_{\mathbb{R}^{3} \times \mathbb{R}^{3}} \frac{|\varphi(x)|^{2}|\varphi(y)|^{2}}{|x-y|} d x d y
$$

if radial symmetry of $\varphi$ is assumed.

In the general case, without restricting to radial functions, the upper bound for the Coulomb energy is given by the well known Hardy-LittlewoodSobolev inequality while lower bounds have been proved only very recently. In particular if one can control suitable homogeneous Sobolev space $\dot{H}^{s}\left(\mathbb{R}^{3}\right)$ the $L^{p}$ lower bound for the Coulomb energy is given by the following inequalities

$$
\|\varphi\|_{L^{p}\left(\mathbb{R}^{3}\right)} \leq C(p, s)\|\varphi\|_{\dot{H}^{s}\left(\mathbb{R}^{3}\right)}^{\frac{\theta}{2-\theta}}\left(\iint_{\mathbb{R}^{3} \times \mathbb{R}^{3}} \frac{|\varphi(x)|^{2}|\varphi(y)|^{2}}{|x-y|} d x d y\right)^{\frac{1-\theta}{4-2 \theta}}
$$

2010 Mathematics Subject Classification: 46E35, 39B62.

Key words and phrases: radial symmetry, sharp emebeddings, Coulomb energy, fractional Sobolev spaces. 
with $\theta=\frac{6-\frac{5}{2} p}{3-p s-p}$. Here the parameters $s>0$ and $1<p \leq \infty$ satisfy

$$
\begin{array}{ll}
p \in\left[\frac{6}{3-2 s}, \frac{2+4 s}{1+s}\right] & \text { if } 0<s<1 / 4, \\
p=\frac{6}{3-2 s}=\frac{2+4 s}{1+s} & \text { if } s=1 / 4, \\
p \in\left[\frac{2+4 s}{1+s}, \frac{6}{3-2 s}\right] & \text { if } 1 / 4<s<3 / 2, \\
p \in\left[\frac{2+4 s}{1+s}, \infty\right) & \text { if } s=3 / 2, \\
p \in\left[\frac{2+4 s}{1+s}, \infty\right] & \text { if } s>3 / 2 .
\end{array}
$$

These bounds have been proved in [3] while the case $s=\frac{1}{2}$ has been first considered in [4]. These bounds follows from a suitable Gagliardo-Nirenberg inequality, see Theorem 2.44 of [1], together with the following well known identity

$$
\iint_{\mathbb{R}^{3} \times \mathbb{R}^{3}} \frac{|\varphi(x)|^{2}|\varphi(y)|^{2}}{|x-y|} d x d y=c\left\|\varphi^{2}\right\|_{\dot{H}^{-1}\left(\mathbb{R}^{3}\right)}^{2} .
$$

We shall underline that in many physical applications involving Sobolev norms and Coulomb energy the radially symmetric assumption of $\varphi$ is natural due to the rotational invariance of energy functionals (see e.g [9] in the context of stability of matter). Our purpose is to see if it is possible to control lower $L^{p}$ norms if one assumes radial symmetry of $\varphi$. In the sequel we use two theorems that are crucial for our improvement in case of radial symmetry. The first is the following pointwise decay for radial functions in $\dot{H}^{s}\left(\mathbb{R}^{d}\right) \cap L_{a}^{q}\left(\mathbb{R}^{d}\right)$, see $[6]$, where $L_{a}^{q}\left(\mathbb{R}^{d}\right)$ is the weighted Lebesgue space with the norm

$$
\|u\|_{L_{a}^{q}\left(\mathbb{R}^{d}\right)}=\left(\int_{\mathbb{R}^{d}}|x|^{a}|u|^{q} d x\right)^{\frac{1}{q}}
$$

Theorem 1.1 (De Nápoli [6]). Let $\varphi$ be a radial function in $\dot{H}^{s}\left(\mathbb{R}^{d}\right) \cap$ $L_{a}^{q}\left(\mathbb{R}^{d}\right)$ with $s>\frac{1}{2}$ and $-(d-1)<a<d(q-1)$, then

$$
|\varphi(x)| \leq C(d, s, q, a)|x|^{-\sigma}\left\|(-\Delta)^{\frac{s}{2}} \varphi\right\|_{L^{2}\left(\mathbb{R}^{d}\right)}^{\theta}\|\varphi\|_{L_{a}^{q}\left(\mathbb{R}^{d}\right)}^{1-\theta}
$$

where $\theta=\frac{2}{2 s q+2-q}, \sigma=\frac{2 a s+2 d s-a-2 s}{2 s q+2-q}$.

Remark 1.1. The strategy of the proof of Theorem 1.1 is based on Fourier representation for radial functions in $\mathbb{R}^{d}$ (identifying the function with its 
profile)

$$
\varphi(x)=(2 \pi)^{\frac{d}{2}}|x|^{-\frac{d-2}{2}} \int_{0}^{\infty} J_{\frac{d-2}{2}}(|x| \rho) \hat{\varphi}(\rho) \rho^{\frac{d}{2}} d \rho
$$

where $J_{\frac{d-2}{2}}$ is the Bessel function of order $\frac{d-2}{2}$. The argument is similar to the one developed in [5] for the pointwise decay of radial function in $\dot{H}^{s}\left(\mathbb{R}^{d}\right)$, i.e to split $\varphi$ into low and high frequency parts. The pointwise decay of the high frequency part of $\varphi$ is controlled by the boundness of Sobolev norm while the decay of low frequency part by the boundness of the weighted Lebesgue norm.

The second theorem is the following lower bound for the Coulomb energy by Ruiz, see [11].

Theorem 1.2 (Ruiz [11]). Given $\alpha>\frac{1}{2}$, there exists $c=c(\alpha)>0$ such that for any measurable $\varphi: \mathbb{R}^{d} \rightarrow \mathbb{R}$ we have

$$
\iint_{\mathbb{R}^{d} \times \mathbb{R}^{d}} \frac{|\varphi(x)|^{2}|\varphi(y)|^{2}}{|x-y|^{d-2}} d x d y \geq c\left(\int_{\mathbb{R}^{d}} \frac{|\varphi(x)|^{2}}{|x|^{\frac{d-2}{2}}(1+|\log | x||)^{\alpha}} d x\right)^{2} .
$$

Let us define

$$
\mathcal{E}^{s}=\left\{\varphi \in \dot{H}_{\text {rad }}^{s}\left(\mathbb{R}^{3}\right) \text { s.t } \iint_{\mathbb{R}^{3} \times \mathbb{R}^{3}} \frac{|\varphi(x)|^{2}|\varphi(y)|^{2}}{|x-y|} d x d y<\infty\right\}
$$

with

$$
\|\varphi\|_{\mathcal{E}^{s}}=\left(\|\varphi\|_{\dot{H}^{s}\left(\mathbb{R}^{3}\right)}^{2}+\left(\iint_{\mathbb{R}^{3} \times \mathbb{R}^{3}} \frac{|\varphi(x)|^{2}|\varphi(y)|^{2}}{|x-y|} d x d y\right)^{\frac{1}{2}}\right)^{\frac{1}{2}}
$$

Following the argument of Ruiz [11] it is easy to show that $\|\cdot\|_{\mathcal{E}^{s}}$ is a norm and $C_{0}^{\infty}\left(\mathbb{R}^{3}\right)$ is dense in $\mathcal{E}^{s}$. In [11] Ruiz proved that for $\mathcal{E}^{1}$ the following continuous embedding

$$
\mathcal{E}^{1} \hookrightarrow L^{p} \quad p \in\left(\frac{18}{7}, 6\right]
$$

The result by Ruiz follows from two steps: first, Theorem 1.2 proves that $\mathcal{E}^{1} \subset \dot{H}_{\text {rad }}^{1}\left(\mathbb{R}^{3}\right) \cap L^{2}\left(\mathbb{R}^{3}, V(x) d x\right)$ where $V(x)=\frac{1}{(1+|x|)^{\gamma}}$ with $\gamma>\frac{1}{2}$, second, a weighted Sobolev embedding for radial function proved by $\mathrm{Su}$, Wang and 
Willem [12] gives the inclusion

$$
\dot{H}_{\text {rad }}^{1}\left(\mathbb{R}^{3}\right) \cap L^{2}\left(\mathbb{R}^{3}, V(x) d x\right) \subset L^{q}\left(\mathbb{R}^{3}\right) \quad q \in\left[\frac{2(4+\gamma)}{4-\gamma}, 6\right]
$$

\section{Main results}

The aim of our paper is to find continuous embeddings and hence better lower bounds for the Coulomb energy assuming radial symmetry when $\frac{1}{2}<$ $s<\frac{3}{2}$. As a particular case we recover the left end-point exponent found by Ruiz [11] when $s=1$.

Theorem 2.1. $\mathcal{E}^{s} \hookrightarrow L^{p}\left(\mathbb{R}^{3}\right)$ continuously for

$$
p \in\left(\frac{16 s+2}{6 s+1}, \frac{6}{3-2 s}\right] \quad \text { if } 1 / 2<s<3 / 2 .
$$

The above result is sharp when $\frac{1}{2}<s \leq 1$ as shown by the following

Theorem 2.2. Let $\frac{1}{2}<s \leq 1$, then the space $\mathcal{E}^{s}$ is not embedded in $L^{p}$ for $p<\frac{16 s+2}{6 s+1}$.

From the continuous embedding for $\mathcal{E}^{s}$ in the radial case it is elementary to derive the scaling invariant lower bounds for the Coulomb energy given by (1.1) for $p \in\left(\frac{16 s+2}{6 s+1}, \frac{6}{3-2 s}\right]$ and $\frac{1}{2}<s<\frac{3}{2}$. Moreover, the best constants in the lower bounds for radially symmetric functions are achieved.

Corollary 2.1. Let $\varphi$ be radially symmetric, then the following scaling invariant inequality holds

$$
\|\varphi\|_{L^{p}\left(\mathbb{R}^{3}\right)} \leq C(p, s)\|\varphi\|_{\dot{H}^{s}\left(\mathbb{R}^{3}\right)}^{\frac{\theta}{2-\theta}}\left(\iint_{\mathbb{R}^{3} \times \mathbb{R}^{3}} \frac{|\varphi(x)|^{2}|\varphi(y)|^{2}}{|x-y|} d x d y\right)^{\frac{1-\theta}{4-2 \theta}}
$$

with $\theta=\frac{6-\frac{5}{2} p}{3-p s-p}$. Here the parameters $s$ and $p$ satisfy

$$
p \in\left(\frac{16 s+2}{6 s+1}, \frac{6}{3-2 s}\right] \quad \text { if } 1 / 2<s<3 / 2 .
$$

Moreover the best constants in these lower bounds are achieved. 


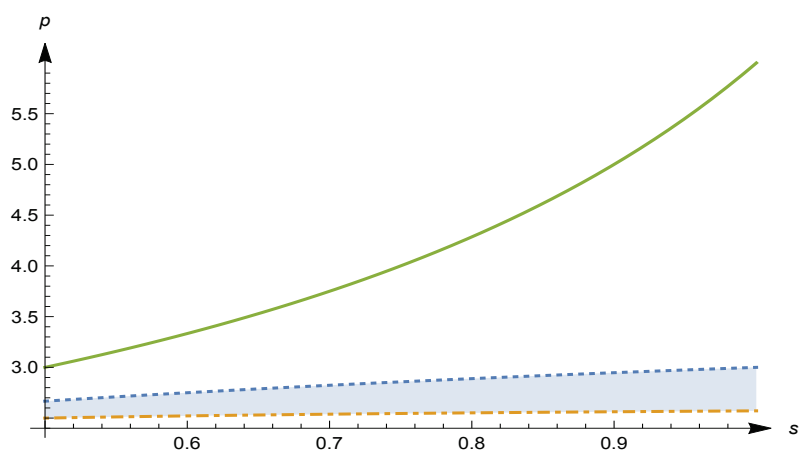

Figure 1: Left endpoint exponent for $p$ in (1.1) and in Theorem 2.1, as a function of $s$ : without radial symmetry the lower bound is $\frac{2+4 s}{1+s}$ (dotted) and for the radially symmetric case is $\frac{16 s+2}{6 s+1}$ (dash-dotted). The bold line plots the right endpoint exponent for $p$ in (1.1) and Theorem 2.1 given by the Sobolev embedding exponent.

Remark 2.1. Notice that for the right endpoint exponent, $p=\frac{6}{3-2 s}$, our inequality is nothing but Sobolev inequality. The existence of maximizers for Sobolev inequality has been proved by Lieb, see e.g. [8].

Remark 2.2. Since our approach is based on Theorem 1.1 and Theorem 1.2, there are no obstructions to study lower bounds for the Coulomb energy with potential $|x-y|^{-(d-2)}$ in dimensions $d>3$ in the radial case. Here we decided to consider only the physically relevant case. We quote [10] for a systematic study in this direction when $s=1$.

In Figure 1 the left endpoint exponent for $p$ in (1.1) and in Theorem 2.1 as a function of $s$ is plotted.

Funding. J.B. is supported by FIRB2012 "Dinamiche dispersive: analisi di Fourier e metodi variazionali", J.B and M.G by GNAMPA2014 "Equazioni di campo non-lineari: solitoni e dispersione".

\section{Proof of Theorem 2.1}

Proposition 3.1. Let $\gamma>\frac{1}{2}$ and $\frac{\gamma}{2}<s<\frac{3}{2}$ then exists $c(\gamma, s)>0$ such that for any $\varphi \in \mathcal{E}^{s}$

$$
\left(\int_{\mathbb{R}^{3}}|x|^{-\gamma}|\varphi|^{2} d x\right) \leq c(\gamma, s)\|\varphi\|_{\mathcal{E}^{s}}^{2} .
$$


Proof. By elementary computation we notice that if $2 s>\gamma$ then

$$
\begin{aligned}
\left(\int_{\mathbb{R}^{3}}|x|^{-\gamma}|\varphi|^{2} d x\right) \leq & R^{2 s-\gamma}\left(\int_{B(0, R)} \frac{|\varphi|^{2}}{|x|^{2 s}} d x\right) \\
& +\frac{(1+R)^{\gamma}}{R^{\gamma}}\left(\int_{B(0, R)^{c}} \frac{|\varphi|^{2}}{(1+|x|)^{\gamma}} d x\right) .
\end{aligned}
$$

Notice that, if $0<s<\frac{3}{2}$, the Pitt's inequality [2] states that

$$
\left(\int_{\mathbb{R}^{3}} \frac{|\varphi|^{2}}{|x|^{2 s}} d \xi\right) \leq c_{s}\left(\int_{\mathbb{R}^{3}}|\hat{\varphi}|^{2}|\xi|^{2 s} d \xi\right)=c_{s}\|\varphi\|_{\dot{H}^{s}\left(\mathbb{R}^{3}\right)}^{2}
$$

where $c_{s}=\pi^{2 s}\left[\frac{\Gamma\left(\frac{3-2 s}{4}\right)}{\Gamma\left(\frac{3+2 s}{4}\right)}\right]^{2}$. Moreover, taking $\gamma>\frac{1}{2}$, by means of the elementary inequality for $\stackrel{4}{x} \neq 0$

$$
\frac{1}{(1+|x|)^{\gamma}} \leq \frac{c(\gamma)}{|x|^{\frac{1}{2}}(1+|\log | x||)^{\gamma}}
$$

and by Ruiz's Theorem 1.2 we have that

$$
\left(\int_{\mathbb{R}^{3}} \frac{|\varphi|^{2}}{(1+|x|)^{\gamma}} d x\right) \leq c(\gamma)\left(\iint_{\mathbb{R}^{3} \times \mathbb{R}^{3}} \frac{|\varphi(x)|^{2}|\varphi(y)|^{2}}{|x-y|} d x d y\right)^{\frac{1}{2}}
$$

and therefore the desired inequality.

Proof of Theorem 2.1. By Proposition 3.1 we have that $\mathcal{E}^{s} \subset L^{2}\left(\mathbb{R}^{3}, V(x) d x\right)$ where $V(x)=|x|^{-\gamma}, \gamma>\frac{1}{2}$. Let us call $p^{*}=\frac{2+4 s}{1+s}$, the left end-point exponent for (1.1). Hölder's inequality assures that for $p<p^{*}$

$$
\begin{aligned}
\int_{B(0,1)}|\varphi|^{p} d x & <\mu(B(0,1))^{\frac{p^{*}-p}{p}}\left(\int_{B(0,1)}|\varphi|^{p^{*}} d x\right)^{\frac{p}{p^{*}}} \\
& \leq C\|\varphi\|_{\dot{H}^{s}\left(\mathbb{R}^{3}\right)}^{\left(\frac{\theta}{2-\theta}\right) p}\left(\iint_{\mathbb{R}^{3} \times \mathbb{R}^{3}} \frac{|\varphi(x)|^{2}|\varphi(y)|^{2}}{|x-y|} d x d y\right)^{\left(\frac{1-\theta}{4-2 \theta}\right) p}
\end{aligned}
$$


with $\theta=\frac{6-\frac{5}{2} p^{*}}{3-p^{*} s-p^{*}}$. On the other hand by Proposition 3.1 and the radial decay given by Theorem 1.1 choosing $a=-\gamma, q=2$ and $d=3$,

$$
\begin{aligned}
\int_{B(0,1)^{c}}|\varphi|^{p} d x & =\int_{B(0,1)^{c}}|\varphi(x)|^{2}|\varphi(x)|^{p-2} d x \\
& \leq\left. C\|\varphi\|_{\dot{H}^{s}\left(\mathbb{R}^{3}\right)}^{\theta(p-2)}\|\varphi\|\right|_{L_{-\gamma}^{2}\left(\mathbb{R}^{3}\right)} ^{(1-\theta)(p-2)} \int_{B(0,1)^{c}}|x|^{-\sigma(p-2)}|\varphi(x)|^{2} d x
\end{aligned}
$$

where $\theta=\frac{1}{2 s}, \sigma=\frac{-2 \gamma s+4 s+\gamma}{4 s}$. Now

$$
\lim _{\gamma \rightarrow \frac{1}{2}} \frac{-2 \gamma s+4 s+\gamma}{4 s}(p-2)=\left(\frac{3 s+\frac{1}{2}}{4 s}\right)(p-2)
$$

and this implies again by Proposition 3.1 that

$$
\int_{B(0,1)^{c}}|\varphi|^{p} d x<+\infty
$$

provided that $\left(\frac{3 s+\frac{1}{2}}{4 s}\right)(p-2)>\frac{1}{2}$, i.e if $p>\frac{16 s+2}{6 s+1}$.

\section{Proof of Theorem 2.2}

The proof of Theorem 2.2 is obtained constructing a counterexample, i.e a function $u$ such that

$$
\begin{aligned}
\|u\|_{\dot{H}^{s}\left(\mathbb{R}^{3}\right)}^{2} & \simeq 1 \\
\iint_{\mathbb{R}^{3} \times \mathbb{R}^{3}} \frac{|u(x)|^{2}|u(y)|^{2}}{|x-y|} d x d y & \simeq 1 \\
\|u\|_{L^{p}\left(\mathbb{R}^{3}\right)}^{p} & \rightarrow+\infty
\end{aligned}
$$

Proof of Theorem 2.2.

The case $s=1$ has been proved by Ruiz [11]. Set $u: \mathbb{R}^{3} \rightarrow \mathbb{R}^{+}$

$$
u(x)= \begin{cases}\varepsilon \frac{S-|| x|-R|}{S} & \text { for }|| x|-R|<S \\ 0 & \text { elsewhere }\end{cases}
$$

where $R>S \gg 1 \gg \varepsilon>0$ will be precised in the sequel. 
We recall, by Ruiz [11, Section 4], that

$$
\iint_{\mathbb{R}^{3} \times \mathbb{R}^{3}} \frac{|u(x)|^{2}|u(y)|^{2}}{|x-y|} d x d y \leq C \varepsilon^{4} S^{2} R^{3}
$$

and

$$
\|u\|_{L^{p}\left(\mathbb{R}^{3}\right)}^{p} \geq C \varepsilon^{p} S R^{2} .
$$

Moreover we have

$$
\|u\|_{\dot{H}^{s}\left(\mathbb{R}^{3}\right)}^{2} \leq C \frac{\varepsilon^{2} R^{2}}{S^{2 s-1}}
$$

The proof of (4.3) is not difficult and it will postponed to Lemma 4.1.

In order to have $\|u\|_{\dot{H}^{s}\left(\mathbb{R}^{3}\right)}^{2} \simeq 1$ we choose $S=\varepsilon^{\frac{2}{2 s-1}} R^{\frac{2}{2 s-1}}$. At this point we have

$$
\iint_{\mathbb{R}^{3} \times \mathbb{R}^{3}} \frac{|u(x)|^{2}|u(y)|^{2}}{|x-y|} d x d y \leq C \varepsilon^{4} \varepsilon^{\frac{4}{2 s-1}} R^{\frac{4}{2 s-1}} R^{3}=C \varepsilon^{\frac{8 s}{2 s-1}} R^{\frac{6 s+1}{2 s-1}}
$$

and we choose $R=\varepsilon^{-\frac{8 s}{6 s+1}}$ to have the Coulomb norm bounded, so

$$
S=\varepsilon^{\frac{2}{2 s-1}} R^{\frac{2}{2 s-1}}=\varepsilon^{\frac{2}{2 s-1}} \varepsilon^{-\frac{8 s}{6 s+1} \frac{2}{2 s-1}}=\varepsilon^{\frac{2-4 s}{(6 s+1)(2 s-1)}}=\varepsilon^{-\frac{2}{6 s+1}} .
$$

We remark that, since $s>1 / 2$, then $8 s>2$ and $R>S$, as required in the definition of $u(x)$.

Concluding, we have

$$
\|u\|_{L^{p}\left(\mathbb{R}^{3}\right)}^{p} \geq C \varepsilon^{p} S R^{2} \simeq \varepsilon^{p-\frac{16 s+2}{6 s+1}}
$$

that diverges for $p<\frac{16 s+2}{6 s+1}$ when $\varepsilon \rightarrow 0$. The claim follows immediately.

Lemma 4.1. Let $u$ be defined in (4.2). Then

$$
\|u\|_{\dot{H}^{s}\left(\mathbb{R}^{3}\right)}^{2} \leq C \frac{\varepsilon^{2} R^{2}}{S^{2 s-1}}
$$


Proof. We want to compute the $\dot{H}^{s}$ norm of $u$ for $s<1$, that is

$$
\|u\|_{\dot{H}^{s}\left(\mathbb{R}^{3}\right)}^{2}=C(s) \int_{\mathbb{R}^{3}} \int_{\mathbb{R}^{3}} \frac{|u(x)-u(y)|^{2}}{|x-y|^{3+2 s}} d x d y .
$$

where $C(s)=2^{2 s-1} \pi^{-\frac{3}{2}} \frac{\Gamma\left(\frac{3+2 s}{2}\right)}{\Gamma(-s)}$. We observe that the set where $u(x)-u(y) \neq$ 0 is contained in the following five subsets of $\mathbb{R}^{3} \times \mathbb{R}^{3}$ :

$$
\begin{aligned}
& A_{1}=\{R-S \leq|y| \leq R+S,|x| \leq R-S\} \\
& A_{2}=\{R-S \leq|y| \leq R+S,|x| \geq R+S\} \\
& A_{3}=\{R-S \leq|x| \leq R+S,|y| \leq R-S\} \\
& A_{4}=\{R-S \leq|x| \leq R+S,|y| \geq R+S\} \\
& A_{5}=\{R-S \leq|x| \leq R+S, R-S \leq|y| \leq R+S\}
\end{aligned}
$$

and, by symmetry, we obtain

$$
\begin{aligned}
\frac{\|u\|_{\dot{H}^{s}\left(\mathbb{R}^{3}\right)}^{2}}{C(s)}= & \iint_{\mathbb{R}^{3}} \frac{|u(x)-u(y)|^{2}}{|x-y|^{3+2 s}} d x d y=2 \iint_{A_{1}} \frac{|u(x)-u(y)|^{2}}{|x-y|^{3+2 s}} d x d y \\
& +2 \iint_{A_{2}} \frac{|u(x)-u(y)|^{2}}{|x-y|^{3+2 s}} d x d y+\iint_{A_{5}} \frac{|u(x)-u(y)|^{2}}{|x-y|^{3+2 s}} d x d y \\
\leq & 2 \int_{R-S \leq|y| \leq R+S} \int_{\mathbb{R}^{3}} \frac{|u(x)-u(y)|^{2}}{|x-y|^{3+2 s}} d x d y .
\end{aligned}
$$

Therefore,

$$
\begin{aligned}
\|u\|_{\dot{H}^{s}\left(\mathbb{R}^{3}\right)}^{2} \leq & C \int_{R-S \leq|y| \leq R+S} \int_{|x-y| \leq S} \frac{|u(x)-u(y)|^{2}}{|x-y|^{3+2 s}} d x d y \\
& +C \int_{R-S \leq|y| \leq R+S} \int_{|x-y| \geq S} \frac{|u(x)-u(y)|^{2}}{|x-y|^{3+2 s}} d x d y \\
\leq & C \int_{R-S \leq|y| \leq R+S} \int_{|x-y| \leq S} \frac{\varepsilon^{2}}{S^{2}} \frac{|x-y|^{2}}{|x-y|^{3+2 s}} d x d y \\
& +C \int_{R-S \leq|y| \leq R+S} \frac{\varepsilon^{2}}{|x-y| \geq S} d x d y
\end{aligned}
$$


using that $|u(x)-u(y)| \leq \sup _{\xi}|\nabla u(\xi)||x-y| \leq \frac{\varepsilon}{S}|x-y|$ in the first term and that $|u(x)| \leq \varepsilon$ in the second term. At this point, with the change of variable $t=x-y$ we get

$$
\begin{aligned}
\|u\|_{\dot{H}^{s}\left(\mathbb{R}^{3}\right)}^{2} & \leq C \varepsilon^{2} \int_{R-S \leq|y| \leq R+S}\left[\int_{|t| \leq S} \frac{1}{S^{2}} \frac{1}{|t|^{\mid+2 s}} d t+\int_{|t| \geq S} \frac{1}{|t|^{3+2 s}} d t\right] d y \\
& \leq C \varepsilon^{2} \int_{R-S \leq|y| \leq R+S}\left[\int_{0}^{S} \frac{1}{S^{2}} \frac{r^{2}}{r^{1+2 s}} d r+\int_{S}^{\infty} \frac{r^{2}}{r^{3+2 s}} d r\right] d y \\
& \leq C \varepsilon^{2} R^{2} S\left[\int_{0}^{S} \frac{1}{S^{2}} r^{1-2 s} d r+\int_{S}^{\infty} r^{-1-2 s} d r\right] \\
& \simeq \varepsilon^{2} R^{2} S\left[\frac{S^{2-2 s}}{S^{2}}+S^{-2 s}\right] \simeq \frac{\varepsilon^{2} R^{2}}{S^{2 s-1}}
\end{aligned}
$$

Proof of Corollary (2.1). Let us consider the following scaling

$$
\varphi_{\lambda}=\lambda^{\frac{3}{p}} \varphi(\lambda x),
$$

such that $\left\|\varphi_{\lambda}\right\|_{L^{p}\left(\mathbb{R}^{3}\right)}=\|\varphi\|_{L^{p}\left(\mathbb{R}^{3}\right)}$ for all $\lambda>0$. From Theorem 2.1 it follows that

$$
\left\|\varphi_{\lambda}\right\|_{L^{p}\left(\mathbb{R}^{3}\right)}^{2} \leq C\left(\left\|\varphi_{\lambda}\right\|_{\dot{H}^{s}\left(\mathbb{R}^{3}\right)}^{2}+\left(\iint_{\mathbb{R}^{3} \times \mathbb{R}^{3}} \frac{\left|\varphi_{\lambda}(x)\right|^{2}\left|\varphi_{\lambda}(y)\right|^{2}}{|x-y|} d x d y\right)^{\frac{1}{2}}\right)
$$

if $p \in\left(\frac{16 s+2}{6 s+1}, \frac{6}{3-2 s}\right]$ and if $1 / 2<s<3 / 2$. By elementary computation one gets

$$
\begin{aligned}
\|\varphi\|_{L^{p}\left(\mathbb{R}^{3}\right)}^{2} \leq C & \left(\lambda^{\frac{6}{p}-(3-2 s)}\|\varphi\|_{\dot{H}^{s}\left(\mathbb{R}^{3}\right)}^{2}\right. \\
& \left.+\lambda^{\frac{6}{p}-\frac{5}{2}}\left(\iint_{\mathbb{R}^{3} \times \mathbb{R}^{3}} \frac{|\varphi(x)|^{2}|\varphi(y)|^{2}}{|x-y|} d x d y\right)^{\frac{1}{2}}\right) .
\end{aligned}
$$

and minimizing the right hand side we get the desired inequality.

The argument to show the existence of maximizers is identical to the one used to show Theorem 2.2 in [3]. We just give a sketch of the proof for reader's convenience. Let us fix $p$ in the set $\left(\frac{16 s+2}{6 s+1}, \frac{6}{3-2 s}\right)$. By homogeneity 
and scaling we can assume that an optimizing sequence $\varphi_{n} \in \mathcal{E}^{s}$ satisfies

$$
\left\|\varphi_{n}\right\|_{\dot{H}^{s}}=\iint_{\mathbb{R}^{3} \times \mathbb{R}^{3}} \frac{\left|\varphi_{n}(x)\right|^{2}\left|\varphi_{n}(y)\right|^{2}}{|x-y|} d x d y=1
$$

and

$$
\left\|\varphi_{n}\right\|_{L^{p}}=C(p, s)+o(1) .
$$

Thanks to inequality (2.1) we can find uniform upper bound on $\left\|\varphi_{n}\right\|_{L^{p_{1}}}$ and $\left\|\varphi_{n}\right\|_{L^{p_{2}}}$ for some $p_{1}<p<p_{2}$. Therefore, by the well known pqr-Lemma (see e.g. Lemma 2.1 in [7])

$$
\inf _{n}\left|\left\{\left|\varphi_{n}\right|>\eta\right\}\right|>0 \text {. }
$$

Now by Lieb's compactness lemma in $\dot{H}^{s}$, see Lemma 2.1 in [3], there exists $\varphi \neq 0$ such that $\varphi_{n} \rightarrow \varphi \in \dot{H}^{s}\left(\mathbb{R}^{3}\right) \cap L^{p}\left(\mathbb{R}^{3}\right)$. Finally, by the non-local Brezis-Lieb lemma for the Coulomb term (see Lemma 2.2 in [3]), and by the Hilbert structure of $\dot{H}^{s}\left(\mathbb{R}^{3}\right)$, i.e that

$$
\left\|\varphi_{n}-\varphi\right\|_{\dot{H}^{s}\left(\mathbb{R}^{3}\right)}^{2}+\|\varphi\|_{\dot{H}^{s}\left(\mathbb{R}^{3}\right)}^{2}=\left\|\varphi_{n}\right\|_{\dot{H}^{s}\left(\mathbb{R}^{3}\right)}^{2}+o(1),
$$

we prove the existence of a maximizer.

\section{Acknowledgements}

The authors thanks Nicola Visciglia for fruitful conversations. The first author thanks also Rupert Frank and Elliot Lieb for interesting discussions around the problem.

\section{References}

[1] H. Bahouri, J.-Y. Chemin, and R. Danchin, Fourier Analysis and Nonlinear Partial Differential Equations, Springer, 2011.

[2] W. Beckner, Pitt's inequality and the uncertainty principle, Proc. Amer. Math. Soc., 123 (1995), no. 6, 1897-1905.

[3] J. Bellazzini, R. L. Frank, and N. Visciglia, Maximizers for GagliardoNirenberg inequalities and related non-local problems, Math. Annalen, (2014), no. 3-4, 653-673.

[4] J. Bellazzini, T. Ozawa, and N. Visciglia, Ground states for semirelativistic Schrödinger-Poisson-Slater energies, arXiv:1103.2649. 
[5] Y. Cho and T. Ozawa, Sobolev inequality with symmetry, Commun. Contemp. Math., 11 (2009), no. 3, 355-365.

[6] P. L. De Nápoli, Symmetry breaking for an elliptic equation involving the Fractional Laplacian, arXiv:1409.7421.

[7] J. Fröhlich, E. H. Lieb, and M. Loss, Stability of Coulomb systems with magnetic fields. I. The one-electron atom., Comm. Math. Phys., 104 (1986), no. 2, 251-270.

[8] E. H. Lieb, Sharp constants in the Hardy-Littlewood-Sobolev and related inequalities, Ann. of Math., 118 (1983), 349-374.

[9] E. H. Lieb and R. Seiringer, The stability of matter in quantum mechanics, Cambridge University Press, Cambridge, 2010.

[10] C. Mercuri, V. Moroz, and J. Van Schaftingen, Groundstates and radial solutions to nonlinear Schrödinger-Poisson-Slater equations at the critical frequency, arXiv: 1507.02837.

[11] D. Ruiz, On the Schrödinger-Poisson-Slater system: behavior of minimizers, radial and nonradial cases, Arch. Ration. Mech. Anal., 198 (2010), no. 1, 349-368.

[12] J. Su, Z.-Q. Wang, and M. Willem, Weighted Sobolev embedding with unbounded and decaying radial potentials, J. Differential Equations, 238 (2007), 201-219.

UNIVERSITÀ DI SASSARI

Via Piandanna 4, 07100 Sassari, Italy

E-mail address: jbellazzini@uniss.it

Dipartimento di Matematica, Università di Pisa

Largo B. Pontecorvo 5, 56100 Pisa, Italy

E-mail address: ghimenti@mail.dm.unipi.it

Department of Applied Physics, Waseda University,

TOKYO 169-8555, JAPAN

E-mail address: txozawa@waseda.jp

Received June 3, 2015 